\title{
Posters presented at Joint Discussion 1
}

Costa, J.E.R., \& Simoes, P.J.A.

Possible thermal emission by relativistic electrons at submillimeter range.

Dasso, S., Matthaeus, W.H., Smith, C.W., \& Milano, L.J.

Observations of solar wind fluctuations: anisotropy properties.

Dieckmann, M.E., Drury, L.O.C., \& Shukla, P.K.

On the ultrarelativistic two-stream instability, electrostatic turbulence and Brownian motion.

Dieckmann, M.E., Shukla, P.K., Eliasson, B., Sircombe, N.J., \& Dendy, R.O. Phase speed of electrostatic waves: The critical parameter for efficient electron surfing acceleration.

\section{Dorman, L.I.}

Great solar energetic particle events: monitoring and forecasting of radiation hazard by using on-line one-min neutron monitor data.

Elyiv, A., Petruk, O., \& Hnatyk, B.

Spectrum of turbulence of the extragalactic magnetic field and IRAS PSCz catalogue.

Gabici, S., \& Blasi, P.

Non-linear shock acceleration and high-energy $\gamma$-rays from clusters of galaxies and filaments.

Gan, W.Q., \& Li, Y.P.

Acceleration region revealed from RHESSI X-ray observations.

Giovannini, G., Giroletti, M., \& Taylor, G.B.

The superluminal giant radio galaxy $1144+35$.

Gopalswamy, N., Yashiro, S., Kaiser, M.L., \& Bougeret, J.-L.

Solar energetic particles and CME-driven shocks.

Hashimoto, T.H., Iye, M.I., \& Aoki, K.A.

Investigation of ionization mechanism of extended narrow line region.

Jones, T.W., \& Kang, H.

Time evolution of cosmic-ray modified MHD shocks

Kang, H., \& Ryu, D.

Particle acceleration at cosmological shock waves.

Karlický, M., \& Bárta, M.

Collapsing magnetic trap as accelerator of electrons in solar flares.

Kashapova, L.K., Zharkova, V.V., Chornogo, S.N., \& Kotrč, P.

Signatures of high energy particle beams in the chromospheric events before the 25 July 2004 flare onset.

Kašparová, J., Kontar, E.P., \& Brown, J.C.

RHESSI survey of photospheric albedo and directivity of solar flare hard X-ray spectra.

Kellogg, E.M., Anderson, C., DePasquale, J., Korreck, K., Nichols, J., Pedelty, J., \& Sokoloski, J.

Hot gas at $10^{7} \mathrm{~K}, \mathrm{Fe} \mathrm{K}-\alpha$ reflection, ongoing non-thermal outbursts, jet formation and $10^{6} \mathrm{~K}$ shocks in the symbiotic binary R Aqr. 
Kotrč, P., \& Kashapova, L.K.

On possible manifestation of non-thermal electrons in the $\mathrm{H} \alpha / \mathrm{H} \beta$ line profile ratio in the June 26, 1999 flare.

Kryvdyk, V.

Acceleration of cosmic rays by stellar collapse.

Kurtanidze, O.M., Fan, J., \& Nikolashvili, M.G.

Photometric observations of $\gamma$-ray loud blazars.

Kurtanidze, O.M., \& Kapanadze, B.Z.

Optical variability of X-ray selected blazars.

Lee, T.-L., \& Hwang, C.-Y.

A new character to distinguish different types of galaxy clusters.

Lopez, E.D.

The Faraday rotation effect in quasar jets.

Marcowith, A.

Turbulence in collisionless shocks.

Mel'nik, V.N., \& Rutkevych, B.P.

Propagation of relativistic electron beams in the plasma of the solar corona.

Nakamura, K., \& Shigeyama, T.

Type Ic supernovae as sources of cosmic rays.

Peterson, B.A., \& Schmidt, B.

Time resolved spectra of the $z=3.7$ GRB 060605 from $+37.1 \mathrm{~min}<\mathrm{t}<+93.1$ min covering $320 \mathrm{~nm}$ to $950 \mathrm{~nm}$

Petri, J.A., \& Kirk, J.G.

Polarisation of high-energy emission in a pulsar striped wind.

Silva, A.V.R., Costa, J.E.R., Castro, C.G.G., Raulin, J.P., Kaufmann, P., Share, G.H., \& Murphy, R.J.

The increasing submillimeter spectral component of intense solar flares.

Štěpán, J., Heinzel, P., Kašparová, J., \& Sahal-Bréchot, S.

Polarization diagnostics of proton beams in solar flares.

Stepanov, A.V., \& Tsap, Yu.T.

Wave-particle interaction and peculiarities of propagation and emission of accelerated particles in solar flares.

Tang, Y., Li, Ch., \& Dai, Y.

The direct electric field acceleration in reconnection current sheet in three GLE events.

Topinka, M., \& Karlický, M.

Simulation of magnetic field dissipation in astrophysical jets.

ud-Doula, A., Townsend, R.H.D., \& Owocki, S.P.

Centrifugal breakout of magnetically confined line-driven stellar winds.

Vandas, M., \& Karlický, M.

Electron acceleration in a wavy shock front.

Varady, M., Karlický, M., \& Kašparová, J.

Return current and the energy deposit in flares.

Zharkova, V.V.

Hard X-ray spectral indices in solar flares as probes of the variations of magnetic field topology during a solar cycle. 